\title{
Regional Differences in the Components of Luminal Water from Rat Gastrointestinal Tract and Comparison with Other Species
}

\author{
Yusuke Tanaka, Toshihiro Hara, Ryoichi Waki, Shunji Nagata. \\ Laboratory of Pharmaceutical Technology, Faculty of Pharmaceutical Sciences, Hiroshima International University, \\ 5-1-1 Hiro-koshingai, Kure, Hiroshima, Japan.
}

Received, March 25, 2012; Revised, June 10, 2012; Accepted, September 9, 2012; Published, September 9, 2012.

\begin{abstract}
Purpose. The bile acids, phospholipids, inorganic ions, and $\mathrm{pH}$ in luminal fluid are important factors for the dissolution and oral absorption of solid drugs. In this study, we evaluated the regional differences in these factors in the rat gastrointestinal (GI) tract. The solubility of griseofulvin, a poorly water-soluble drug, in the luminal fluid in each segment was also measured. In addition, the data from rats were compared with those from other species published previously to evaluate the species differences in the composition of luminal fluid. Methods. Rat abdomen was opened and residual water was sampled from each region of GI tract to measure the various components concentrations. Results. The total bile acid concentration was about 2 times higher in the lower than upper jejunum, and phospholipids were much higher in the upper and lower jejunum. The solubility of griseofulvin in the lower jejunal fluid (153-260 $\mu \mathrm{g} / \mathrm{mL}$ ) was about 1.5-2 times higher than those in the upper jejunal fluid $(99-146 \mu \mathrm{g} / \mathrm{mL})$. The regional differences in inorganic ions and $\mathrm{pH}$ were also observed. As for species differences, the total bile acid and phospholipid concentration in rats GI tract were much higher than those of dogs and humans. Conclusion. The regional and species differences of the components in the GI fluid should be useful to predict dissolution and oral absorption of solid drugs.
\end{abstract}

This article is open to POST-PUBLICATION REVIEW. Registered readers (see "For Readers") may comment by clicking on ABSTRACT on the issue's contents page.

\section{INTRODUCTION}

More than $60 \%$ of marketed drugs are used as oral products. Dissolution is the first step in oral absorption of solid drugs such as tablets and capsules. Bile acid and phospholipid concentrations in the gastrointestinal (GI) tract are important factors that influence the solubility, dissolution rate and oral absorption of drugs due to micellization (1-3). The oral absorption of low-solubility drugs categorized into Biopharmaceutics Classification System (BCS) class II is improved by the secretion of bile in the GI tract, which increases their solubility and dissolution rate $(\mathbf{4}, \mathbf{5})$. Inorganic ion concentrations and $\mathrm{pH}$ also significantly influence the stability of nanoparticles and the degree of supersaturation in several formulations, such as solid dispersions and amorphous forms, (6-9) and it determines the fraction of the drug absorbed $(\mathrm{Fa})$. Although the upper region of the small intestine is generally considered to have a higher capacity for drug absorption, the lower regions of the GI tract such as the lower jejunum and ileum become the main regions for drug absorption when a drug is not absorbed sufficiently in the upper region (10). Prediction and assessment of human oral absorption of poorly soluble candidates at the preclinical phase is very important for avoiding a failure during the development of oral products. Currently, various simulated intestinal fluids, including bile acids and phospholipids, have been developed to predict and/or to assess drug dissolution in the GI tract and/or oral absorption from the GI membrane (11-14). However, these simulated intestinal fluids are prepared according to the composition of upper jejunal fluid in humans and dogs. The bile acids, phospholipids, and inorganic ion concentrations may differ between the upper and lower regions of the GI tract, because they are secreted as bile from the gall bladder into the duodenum and transit to the lower region following absorption from the GI membrane. In addition, their concentrations may change according to the fluid volume in each region of the GI tract. Therefore, evaluating the dissolution of drugs not only in the upper jejunum but also in the lower region of the GI tract is very important for increasing the accuracy of predicting the oral absorption of drugs, particularly nanoparticles, some formulations that induce supersaturation, and poorly water-soluble drugs.

\footnotetext{
Corresponding Author: Dr. Y. Tanaks, Laboratory of Pharmaceutical Technology, Faculty of Pharmaceutical Sciences, Hiroshima International University, 5-1-1 Hiro-koshingai, Kure, Hiroshima, Japan. E-mail: y-tanaka@ps.hirokoku-u.ac.jp
} 
Although some authors have reported the concentrations of bile acids, phospholipids, and inorganic ions in the upper jejunum, (15-17) information regarding their concentrations in the lower part of the GI tract is very limited.

Understanding the pharmacokinetics of drugs after oral administration is essential for drug development. At present, animals including mice, rats, dogs, and monkeys are used to evaluate pharmacokinetics of new drug candidates during preclinical studies, and the data are used to judge the safety and effectiveness in humans prior to clinical studies. However, it is well known that there are large differences in oral bioavailability between species. Species differences exist throughout the process of oral absorption of drugs from the intestinal lumen to the systemic circulation, including the dissolution of a drug in the GI tract, permeation from the GI membrane, and metabolism in the small intestine and liver. Although species differences in drug permeation and metabolism have been widely investigated, (18-21) there is little information about the species differences between rats and other animals regarding the composition of luminal water and its influence on drug dissolution.

In this study, we evaluated the regional differences in the total bile acid, phospholipid and inorganic ion concentrations, and $\mathrm{pH}$, in the rat GI tract. The solubility of griseofulvin, a drug with poor water solubility, in the water in each segment was also measured. In addition, the data from rats were compared with those from other species obtained in previous studies to evaluate the species differences in the composition of luminal water.

\section{MATERIALS AND METHODS}

\section{Materials}

Griseofulvin and chloramphenicol were purchased from WAKO Pure Chemical Industries, Ltd. (Osaka, Japan). All other reagents were analytical-grade commercial products.

\section{Sampling of water from the stomach and small intestine \\ Male Wistar rats were purchased from Japan SLC (Hamamatsu, Japan). Rats weighing about $200 \mathrm{~g}$ were fasted overnight prior to the experiments. After oral administration of $1 \mathrm{~mL}$ of ultrapure water (milli-Q), rats were sacrificed at $10-15 \mathrm{~min}$ intervals over a $75 \mathrm{~min}$ period. The abdomen was opened immediately to collect a sample of luminal water from each segment of the GI tract by using a micropipette and a sponge to wipe the surface of the GI membrane. The sponge was then weighed and the amount of water was calculated by assuming a relative density of water equal to 1. 5.0 $\mu \mathrm{g} / \mathrm{mL}$ chloramphenicol solution was added to each}

sample to be extracted from sponge and to prevent microbial growth, and the samples were stored at $-30^{\circ} \mathrm{C}$ prior to quantification of various components in the samples. The GI segments used in this study were stomach, upper jejunum (from a site 3 to 23 $\mathrm{cm}$ position distal to the stomach) and lower jejunum (from a site 10 to $30 \mathrm{~cm}$ position proximal to the caecum).

The total bile acids, phospholipids and inorganic ions as well as the saturated solubility of griseofulvin were measured using samples obtained from the same rats.

All procedures were performed in accordance with the Guidelines for the Care and Use of Laboratory Animals of the Committee for Animal Experiments of Hiroshima International University and the Japanese Association for Laboratory Animal Science. The approval has been received.

\section{Measurement of the $\mathrm{pH}$ values of the luminal water samples}

Following collection of the luminal water samples was collected from rat GI tract into micro plastic tubes, their $\mathrm{pH}$ values were readily measured using pH spear (Nikko Hansen \& Co., Ltd., Osaka, Japan).

\section{Measurement of inorganic ion concentrations in the luminal water samples \\ The concentrations of $\mathrm{Na}^{+}, \mathrm{K}^{+}, \mathrm{Ca}^{2+}, \mathrm{Cl}^{-}, \mathrm{PO}_{4}^{-}$, and $\mathrm{SO}_{4}{ }^{-}$were measured using an ion chromatography system (DX-AQ1110; Dionex Co., Ltd., Osaka, Japan). DIONEX IonPac CS12 and AS12A were used as the columns for cations and anions, respectively. The mobile phase compositions were $20 \mathrm{mM}$ methanesulfonic acid for cations and $2.7 \mathrm{mM} \mathrm{Na} \mathrm{CO}_{3}$ and $0.3 \mathrm{mM}$ $\mathrm{NaHCO}_{3}$ for anions.}

\section{Quantitative determination of total bile acids}

The concentration of total bile acids in rat luminal water was determined by colorimetric measurement of total $3 \alpha$-hydroxy bile acids by using a Total Bile Acid Test Wako (WAKO Pure Chemical Industries, Ltd.). Because of the high bile acid level in the luminal water, the samples were diluted with ultrapure water to a concentration within the calibration curve for the kit.

\section{Quantitative determination of phospholipids}

Phospholipids including choline such as lecithin, lysolecithin and sphingomyelin were quantified by enzymatic colorimetric determination of choline using an EnzyChromTM phospholipid assay kit (EPLP-100; BioAssay Systems, Hayward, USA). 
Measurement of the saturated solubility of griseofulvin in the water samples from each segment of the rat GI tract.

Excess griseofulvin was suspended in each luminal water sample and was vortexed sufficiently. Then, each sample was shaken in an incubator at $37^{\circ} \mathrm{C}$ for $3 \mathrm{hr}$. The suspension was filtered through a $0.65 \mu \mathrm{m}$ hydrophilic polyvinylidene fluoride membrane (Millipore Corporation, USA). The supernatant was analyzed by HPLC.

Solubility measurement of griseofulvin in fasted-state simulated intestinal fluid.

The saturated solubility of griseofulvin was determined after $24 \mathrm{hr}$ of equilibration in $1 \mathrm{~mL}$ fasted-state simulated $\mathrm{dog}$ and human intestinal fluid $\left(\mathrm{FaSSIF}_{\mathrm{dog}}\right.$ and $\left.\mathrm{FaSSIF}_{\text {human }}\right)$ in an incubator at $37^{\circ} \mathrm{C}(\mathbf{1 1}, \mathbf{1 2}, \mathbf{2 2})$. Excess griseofulvin was suspended in each FaSSIF sample and vortexed, and aqueous samples were filtered through a 0.45 $\mu \mathrm{m}$ cellulose membrane after $24 \mathrm{hr}$. The first 0.6 $\mathrm{mL}$ was discarded to avoid loss of griseofulvin from the sample due to adsorption. The remainder of the sample was diluted with distilled water.

\section{HPLC analysis for griseofulvin}

The griseofulvin solubility in each luminal water sample was determined using an HPLC system composed of an HPLC pump (LC-20AD; Shimadzu Corporation, Kyoto, Japan) and a UV detector (SPD-20A; Shimadzu Corporation, Kyoto, Japan). An analytical column, Zorbax Eclipse XDB-C18 (2.1 $\times 50 \mathrm{~mm}$, I.D., $5 \mu \mathrm{m}$, Agilent Technologies), was used at $40{ }^{\circ} \mathrm{C}$. The mobile phases consisted of $50 \mathrm{mM}$ phosphate buffer (pH4.0) and acetonitrile in a ratio of $1: 1(\mathrm{v} / \mathrm{v})$. Griseofulvin was detected at $293 \mathrm{~nm}$. The coefficient of variation (CV) of griseofulvin standards in the mobile phase was 3.14 . The squared correlation coefficients of griseofulvin were $>0.9973$.

\section{STATISTICAL ANALYSIS}

Statistical differences were assessed applying $F$-test and the Student's $t$-test at $p$ of 0.05 using SPSS Student Version (TokyoTosho Co., Ltd., Japan).

\section{RESULTS}

\section{Determination of $\mathrm{pH}$ in the luminal water samples}

Fig. 1 shows the changes in $\mathrm{pH}$ in each region of the GI tract with time. The $\mathrm{pH}$ in the stomach 10 min after oral administration of $1 \mathrm{~mL}$ ultrapure water was about 1.9 and gradually decreased to 1.4 with time. This may be due to gastric empting of orally ingested water. In contrast, the samples collected from the upper and lower jejunum after
$10 \mathrm{~min}$ had $\mathrm{pH}$ values of about 6.7 and 6.9 and the $\mathrm{pH}$ values gradually increased to 7.0 and 7.4, respectively. The $\mathrm{pH}$ values in the lower jejunum were slightly higher than those in the upper jejunum at the all times.

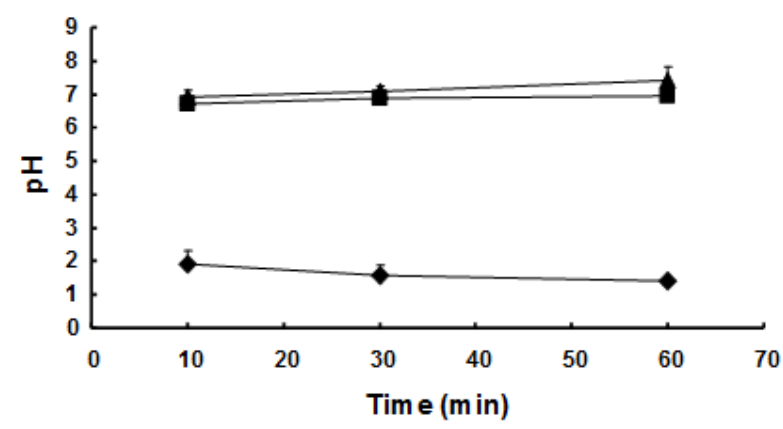

Figure 1. $\mathrm{pH}$ of gastric and intestinal fluid in rat GI tract. The results are expressed as the mean \pm S.D $(n=5-7)$.

$\boldsymbol{\bullet}$, stomach; $\boldsymbol{\square}$, upper jejunum; $\boldsymbol{\Delta}$, lower jejunum.

Inorganic ion concentrations in stomach and upper and lower jejunum

Changes in the concentrations of $\mathrm{Na}^{+}, \mathrm{K}^{+}, \mathrm{Ca}^{2+}$, $\mathrm{Cl}^{-}, \mathrm{PO}_{4}^{-}$, and $\mathrm{SO}_{4}^{-}$within each region are shown in Fig. 2 and Fig. 3. No inorganic ions were detected in the ultrapure water used for oral administration (data not shown). Although $\mathrm{Cl}^{-}$was detected in the $5 \mu \mathrm{g} / \mathrm{mL}$ chloramphenicol solution used for dilution of samples and prevention of microbial growth, the concentration was negligible (0.0013 mM).

The average of $\mathrm{Na}^{+}$concentrations between 10 and $75 \mathrm{~min}$ were $52 \mathrm{mM}$ in stomach, $113 \mathrm{mM}$ in upper jejunum and $153 \mathrm{mM}$ in lower jejunum; therefore, $\mathrm{Na}^{+}$was the dominating ion in the upper and lower jejunal fluid. The dominating ion in the gastric fluid was $\mathrm{Cl}^{-}$, with a mean concentration of $98 \mathrm{mM}$ between 10 and $75 \mathrm{~min}$. The mean concentration of $\mathrm{K}^{+}$between 10 and 75 min was $18 \mathrm{mM}, 51 \mathrm{mM}$ and $42 \mathrm{mM}$ in stomach, upper jejunum, and lower jejunum, respectively. The $\mathrm{K}^{+}$ concentration in the stomach was lower than those in the upper and lower jejunum. The $\mathrm{Ca}^{2+}$ concentration in the lower jejunum $(8 \mathrm{mM})$ was about 4-5 times higher compared with those in stomach $(2 \mathrm{mM})$ and upper jejunum $(1.6 \mathrm{mM})$. Although $\mathrm{SO}_{4}{ }^{-}$concentration was about $3.4 \mathrm{mM}$ in the upper jejunum, $\mathrm{SO}_{4}{ }^{-}$was hardly detected in the stomach and lower jejunum. The mean concentration of $\mathrm{PO}_{4}{ }^{-}$between 10 and 75 min was $3 \mathrm{mM}, 24 \mathrm{mM}$ and $23 \mathrm{mM}$ in stomach, upper jejunum, and lower jejunum, respectively. Large regional differences in the inorganic ion concentrations measured in this study were observed. This may be due to differences in the processes, such as secretion, reabsorption and transit rate, among inorganic ions in each region. 

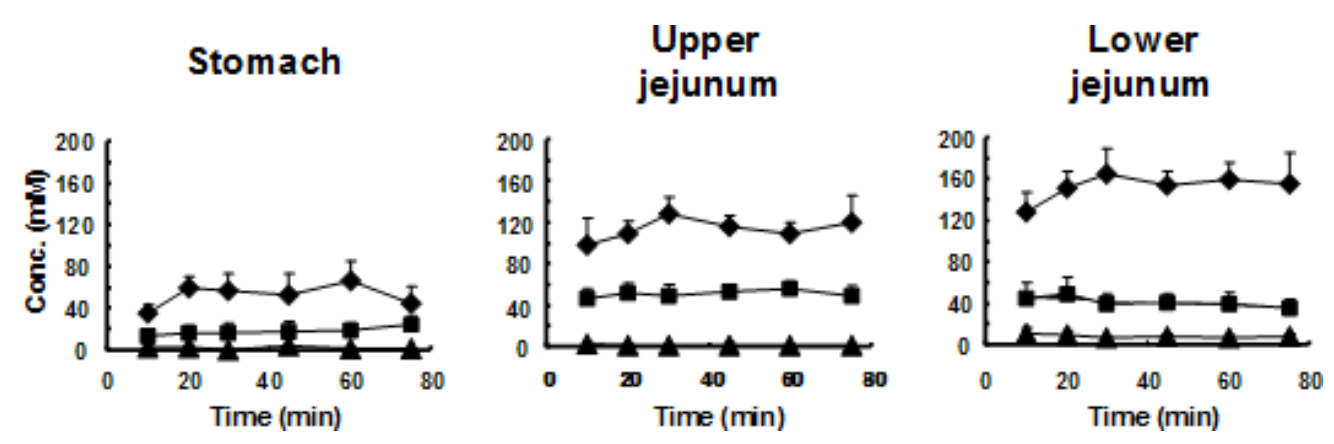

Figure 2. Inorganic cation concentrations in stomach and upper and lower jejunum. The results are expressed as the mean \pm S.D $(\mathrm{n}=4-9) . \bullet, \mathrm{Na}^{+} ; \boldsymbol{\square}, \mathrm{K}^{+} ; \boldsymbol{\Delta}, \mathrm{Ca}^{2+}$
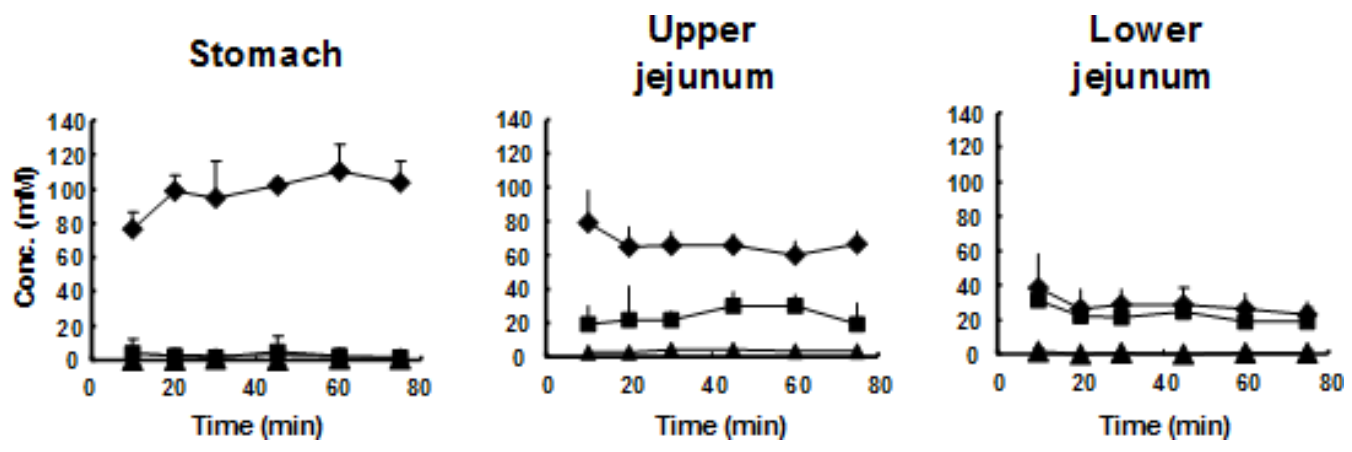

Figure 3. Inorganic anion concentrations in stomach and upper and lower jejunum. The results are expressed as the mean \pm S.D $(\mathrm{n}=4-9)$. $\bullet, \mathrm{CI}^{-} ; \mathbf{\square}, \mathrm{PO}^{4-} ; \boldsymbol{\Lambda}, \mathrm{SO}^{4-}$

Total bile acid concentration in each region

The total bile acid concentrations in each region over time are shown in Fig. 4. The average concentration of total bile acid at each time point was $51 \mathrm{mM}$ in the upper jejunum. The concentration in the lower jejunum was about 2 times higher than that in the upper jejunum. Hagio et al. quantified the total bile acid concentration in the intestinal contents composed of luminal water and foods of fed rats by LC/ESI-MS (23). They observed a similar tendency for the total bile acid concentration to be higher in ileum $(15-25 \mu \mathrm{mol} / \mathrm{g})$ than jejunum $(9-17 \mu \mathrm{mol} / \mathrm{g})$. Assuming that $1 \mathrm{~g}$ of the contents has a volume of $1 \mathrm{~mL}$, the total bile acid concentrations measured by Hagio et al. (9-17 $\mathrm{mM}$ in jejunum and 15-25 $\mathrm{mM}$ in ileum) seem lower compared to our data $(51 \mathrm{mM}$ in upper jejunum and $102 \mathrm{mM}$ in lower jejunum). This may be because the total bile acids were diluted by foods within the rat small intestine. In the stomach, a low concentration of total bile acid was observed. The bile acid in the stomach is derived from the reflux of duodenal fluid. The total bile acid concentrations at $10 \mathrm{~min}$ in the upper and lower jejunum were lower than at the other time points, indicating that ingested water reached these regions by about 10 min.

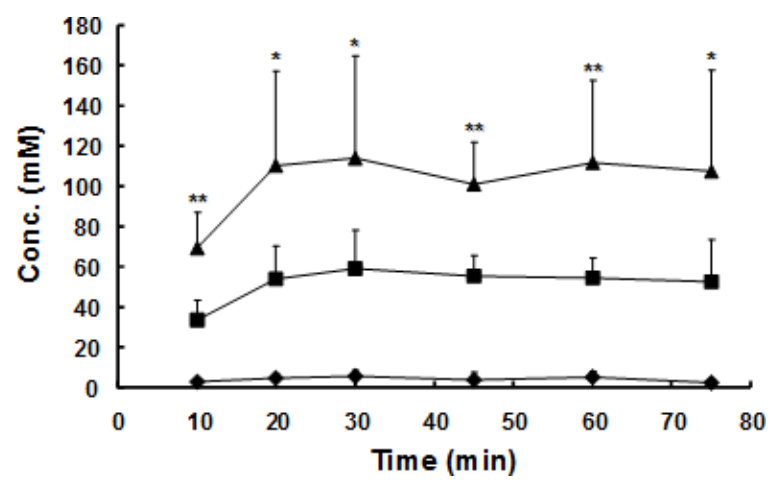

Figure 4. The total bile acid concentration in each GI segment. The results are expressed as the mean \pm S.D (n = 5-9). $\boldsymbol{\bullet}$, stomach; $\boldsymbol{\square}$, upper jejunum; $\boldsymbol{\Delta}$, lower jejunum. $\quad * * \mathrm{P}<0.01 ; \quad * \mathrm{P}<0.05, \quad$ compared to the corresponding parameters of upper jejunum. 


\section{Luminal concentration of phospholipids in each} region

The concentration of phospholipids in the upper jejunum ranged from 1.5 to $5.5 \mathrm{mM}$ until $75 \mathrm{~min}$ after oral administration of $1 \mathrm{~mL}$ of ultrapure water (Fig. 5). However, phospholipids were hardly detected in the lower jejunum. This may be due to the degradation of phospholipids by phospholipase in the intestinal fluid before phospholipids secreted from the sphincter of Oddi to the duodenum reach the lower jejunum.
Saturated solubility of griseofulvin in the luminal water samples

Fig. 6 shows the saturated solubility of griseofulvin in each luminal water sample. The griseofulvin concentrations in gastric fluid were 21.9-27.1 $\mu \mathrm{g} / \mathrm{mL}$, and the griseofulvin concentrations in the luminal water of the lower jejunum (153-260 $\mu \mathrm{g} / \mathrm{mL}$ ) became about twice those in the upper jejunum $(99-146 \mu \mathrm{g} / \mathrm{mL})$.

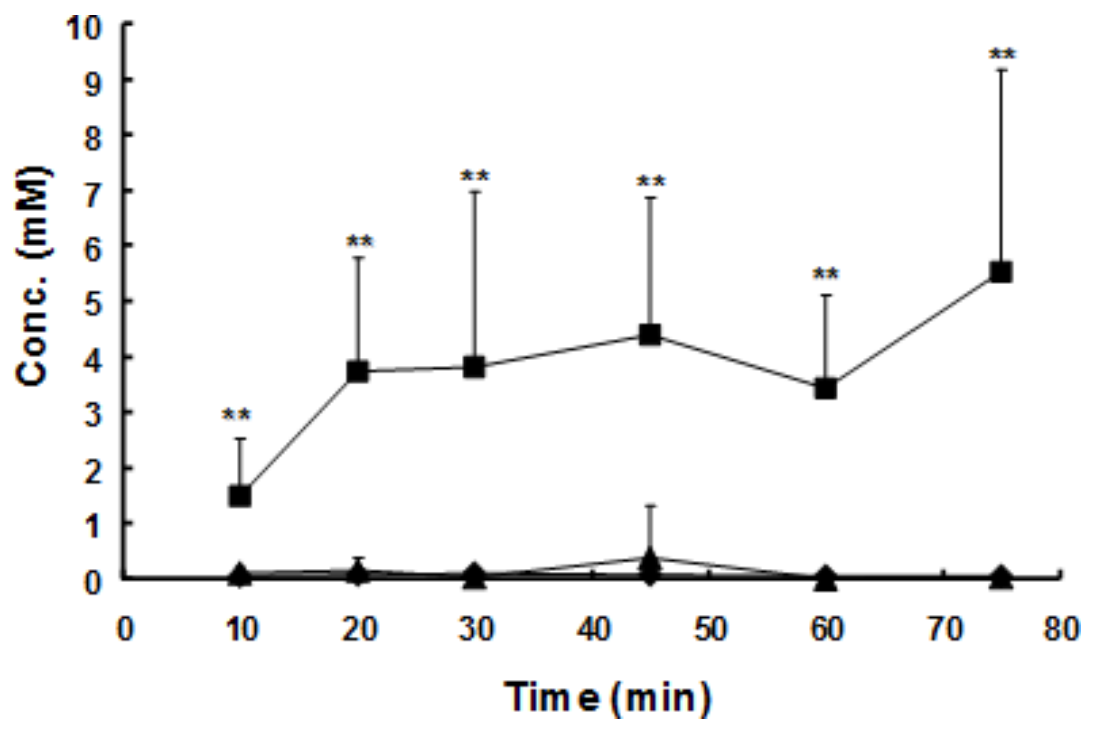

Figure 5. The phospholipids concentration in each GI segment. The results are expressed as the mean \pm S.D $(n=6-9)$. $* * \mathrm{p}<0.01$ compared to the corresponding parameters of lower jejunum. $\bullet$, stomach; $\mathbf{\square}$, upper jejunum; $\boldsymbol{\Delta}$, lower jejunum.

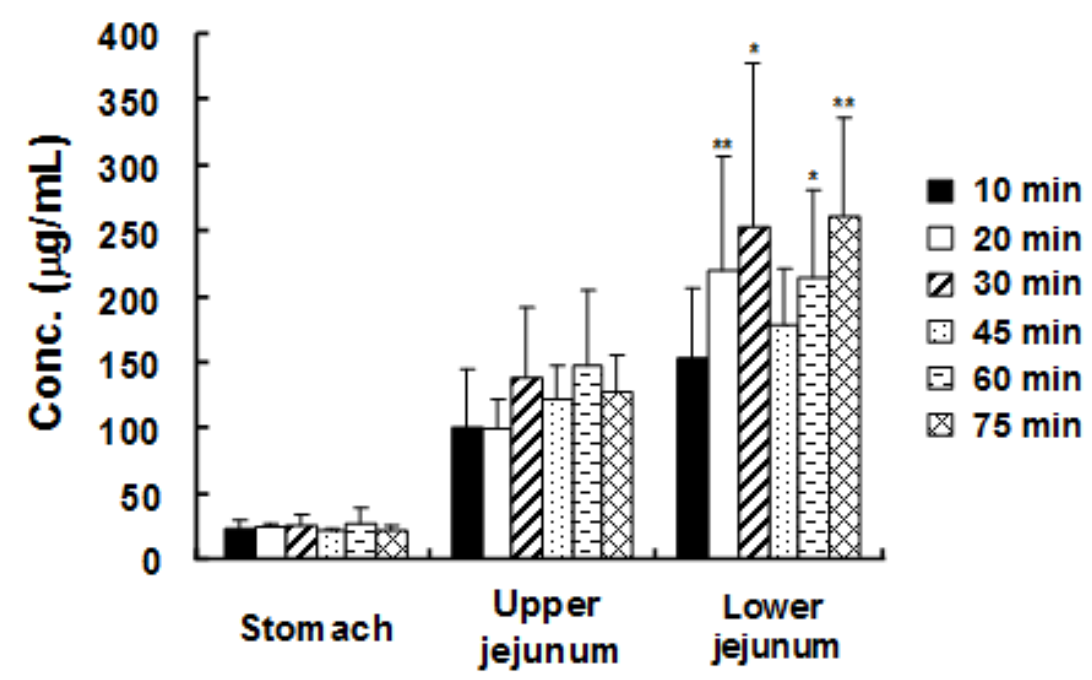

Figure 6. Saturated solubility of griseofulvin in each luminal water at each time point after oral administration of water. The results are expressed as the mean \pm S.D $(n=5-9)$. ${ }^{*} \mathrm{p}<0.01 ;{ }^{*} \mathrm{P}<0.05$, compared to the corresponding parameters of upper jejunum. 


\section{DISCUSSION}

\section{Regional differences}

In this study, to consider the regional differences in the dissolution and oral absorption of drugs, the compositions of luminal water sampled from stomach, upper jejunum, and lower jejunum were investigated.

Large regional differences in the inorganic ion concentrations measured in this study were observed (Fig. 2 and 3). High ion strength sometimes reduces the stability of nanoparticles and the supersaturation level of a drug stabilized by surfactants or polymers in solution, and can lead to aggregation of nanoparticles and precipitation of drugs out of solution (6-9). Ion strengths (I) calculated from the inorganic ion concentrations using the equation of $\mathrm{I}=0.5 \sum \mathrm{CZ}^{2}(\mathrm{C}$ : molarities, $\mathrm{Z}$ : valency of each ion) were $0.087,0.130$, and 0.133 in stomach, upper jejunum and lower jejunum, respectively. Reduced stability of these formulations might tend to be induced in the small intestine more than in the stomach. However, regional differences may not exist between upper and lower jejunum.

$\mathrm{Na}^{+}$-dependent transporters such as SGLT1 $\left(\mathrm{Na}{ }^{+}\right.$-glucose cotransporter), ASBT (apical sodium-dependent bile acid transporter), and SVCTs (sodium-dependent vitamin C transporters) are expressed in the small intestine (24-26). The absorption rates of substrates by these transporters depend on the $\mathrm{Na}^{+}$concentration on the apical side (27). SGLT1 mainly participates in the absorption of nutritional D-glucose and D-galactose across the apical membrane of epithelial cells in the small intestine (28). Otake et al. synthesized glucose-quinoline derivatives and improved the permeability of the quinoline on rat intestinal brush-border membrane vesicles (BBMVs) by utilizing SGLT1 as a target (29). When predicting the total uptake of drugs or substrates utilizing such transporters in vivo, adjustment of the $\mathrm{Na}^{+}$ concentration to in vivo levels may increase the accuracy of prediction (the concentrations of $\mathrm{Na}^{+}$ in the upper and lower jejunum of rats are $110 \mathrm{mM}$ and $150 \mathrm{mM}$, respectively).

The total bile acid concentration was lower in upper jejunum (about $50 \mathrm{mM}$ ) than in lower jejunum (about $100 \mathrm{mM}$ ) (Fig. 4). The absorption rate of bile acid from the small intestine is slower than that of luminal water, which may lead to the condensation of bile acids in the lower jejunum. Masaoka et al. reported a similar phenomenon regarding the luminal concentration of atenolol (a low-permeability drug) (30). According to this report, following oral administration of atenolol, the peak luminal concentrations in the upper and lower jejunum were 1.2-1.5 times higher than the dose concentration.
Phospholipids are secreted in bile from the sphincter of Oddi to the duodenum (31). The phospholipid concentration was about $3.7 \mathrm{mM}$ in upper jejunum, but phospholipids were scarcely detected in lower jejunum (Fig. 5). In this study, the cholines enzymatically released from phospholipids including lecithin, lysolecithin, and sphingomyelin were quantified to determine the phospholipid concentration. Phospholipase D and alkaline phosphatase hydrolyze the phosphate esters in these phospholipids into phosphatidic acid and choline, which are abundant in intestinal fluid $(\mathbf{3 2}, \mathbf{3 3})$. Therefore, the phospholipid concentration might be decreased by these enzymes during transit to the lower jejunum.

The saturated solubility of griseofulvin in the lower jejunum was about 1.5-2 times higher than that in the upper jejunum at each time point (Fig. 6). This might be because the total bile acid concentration in the lower jejunum was about twice that in the upper jejunum. Therefore, more griseofulvin was solubilized in the luminal water obtained from the lower jejunum. However, the concentration of phospholipids, which also have surfactant properties, was higher in the upper jejunum than in the lower jejunum. The contribution of phospholipids to the solubility of griseofulvin may be quite small, because the total bile acid concentration was much higher (about 50 $\mathrm{mM}$ in upper jejunum and $100 \mathrm{mM}$ in lower jejunum) compared with phospholipids (about 3.7 $\mathrm{mM}$ in upper jejunum and $0.12 \mathrm{mM}$ in lower jejunum). This result clearly indicates that dissolution of low-solubility drugs, such as those in BCS class II, may be enhanced in the lower region of the GI tract compared to the upper region. Therefore, considering that the mean residence time of drugs in the lower region of the GI tract is longer than in the upper region $(\mathbf{1 0}, \mathbf{3 0})$, evaluation of the dissolution in the lower region is more important to increase the accuracy of predicting the oral absorption of low-solubility drugs. Fotaki et al. and Jantratid et al. used dissolution media simulating human intestinal fluid of the proximal colon and ileum to evaluate the dissolution of drugs in these regions $(\mathbf{3 4}, \mathbf{3 5})$. Although these media were prepared on the basis of information such as the osmolality, buffer capacity, and ionic strength of intestinal fluid, it might be assumed that bile acids and phospholipids were completely absorbed or degraded in these regions, and the composition of inorganic ions was not considered. Sugano et al. suggested that the oral absorption of certain low-permeability drugs, such as those in BCS class III, decreases after ingestion of foods (negative food effect) (36). This is because dissolved drugs are taken up into micelles formed by bile acids and phospholipids, this reduces the free concentration of the drugs in the intestinal fluid. In rats, it is 
considered that the free concentration of low-permeability drugs in the lower region of the small intestine may become much lower because of the higher concentration of bile acids in the lower jejunum; furthermore, the surface area of the upper jejunum is larger compared to the lower region of the small intestine. Accordingly, in contrast to poor-solubility drugs, it is possible that the absorption rate of low-permeability drugs from the GI membrane in the lower region might be much lower than in the upper part. To predict oral absorption of BCS class III drugs, it is important to evaluate the permeability of low-permeability drugs based on their free concentration in the lower region of the GI tract. After oral administration of drugs categorized into BCS class II and class III, most of the dose would reach the lower region of the GI tract due to their slow dissolution and absorption rate $(\mathbf{1 0}, \mathbf{3 0})$, so the information about the regional differences of the components in the GI fluid should be very useful to consider the oral absorption of these drugs.

\section{Species differences}

The concentrations of each component in the rat GI tract were compared with those in other species published previously to evaluate species differences in the components of the upper jejunum under fasted conditions.

It is reported that the $\mathrm{Na}^{+}, \mathrm{K}^{+}, \mathrm{Ca}^{2+}$ and $\mathrm{Cl}^{-}$ concentrations in upper jejunal fluid are 142, 5.4, 126 , and $0.5 \mathrm{mM}$ in humans, and the order of the concentrations is $\mathrm{Na}^{+}>\mathrm{Cl}^{-}>\mathrm{K}^{+}>\mathrm{Ca}^{+}$(15). The same order was observed in rats, and the ionic strength of rat jejunal fluid (0.13) was also comparable with humans (0.139) (15). No species differences in inorganic ions were observed in rats and humans.

The total bile acid concentrations in the upper jejunum are about $1.52-2.9 \mathrm{mM}$ in humans and 2.41-9.39 $\mathrm{mM}$ in dogs under fasted conditions (15-17). Phospholipid concentrations are less than 0.2 and $0.5 \mathrm{mM}$ in humans and dogs under fasted conditions, respectively, although one outlier was observed in both species $(0.3 \mathrm{mM}$ in humans and $8.12 \mathrm{mM}$ in dogs) (17). These concentrations were much higher in rats (about $51 \mathrm{mM}$ for bile acids and $3.7 \mathrm{mM}$ for phospholipids, averaged between 10 and $75 \mathrm{~min}$ ). One of the reasons for this is that the rat does not possess a gall bladder, and so bile constantly excretes into the duodenum even in fasted conditions.

Fasted-state simulated intestinal fluids for humans and dogs FaSSIF $_{\text {human }}$ and FaSSIF $_{\text {dog, }}$, containing $3 \mathrm{mM}$ and $5 \mathrm{mM}$ of sodium taurocholate, and $0.75 \mathrm{mM}$ and $1.25 \mathrm{mM}$ of lecithin, respectively) have been developed according to the composition of components in upper jejunal fluid to simulate drug solubility and dissolution in the animal small intestine $(\mathbf{1 1}, \mathbf{1 2}, \mathbf{1 4})$. There are several reports that the dissolution and oral absorption of several drugs in humans and dogs were well simulated using these dissolution media $(4,37,38)$, and FaSSIF $_{\text {human }}$ and FaSSIF $_{\text {dog }}$ were used to simulate the solubility of griseofulvin in the upper intestinal fluid of humans and dogs to compared with that of rats in this study (Fig. 7).

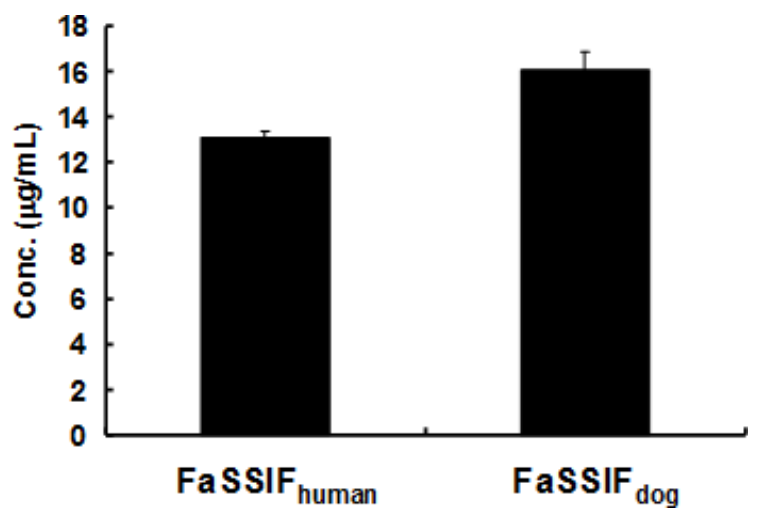

Figure 7. Saturated solubility of griseofulvin in FaSSIF human and $\mathrm{FaSSIF}_{\mathrm{dog}}$. The results are expressed as the mean \pm S.D $(n=6)$.

The solubility of griseofulvin in FaSSIF $_{\text {human }}$ (13.1 $\mu \mathrm{g} / \mathrm{mL})$ and $\mathrm{FaSSIF}_{\mathrm{dog}}(16.1 \mu \mathrm{g} / \mathrm{mL})$ was much lower than that in jejunal fluid in rats $(122.4 \mu \mathrm{g} / \mathrm{mL}$, average at $10-75 \mathrm{~min})$. The total bile acid and phospholipid concentrations were much higher in upper jejunal fluid in rats than in $\mathrm{FaSSIF}_{\text {human }}$ and $\mathrm{FaSSIF}_{\mathrm{dog}}$, and therefore, more griseofulvin was incorporated into the mixed micelles in the upper jejunal fluid from rats. It is strongly indicated that the dissolution of poorly soluble drugs in the rat upper jejunum is much higher compared to humans and dogs, and this may lead to the large difference in the fraction absorbed $(\mathrm{Fa})$ among these species. Takano et al. have established a prediction method for the oral absorption behavior of poorly water-soluble drugs by elucidating the rate-limiting steps of oral absorption $(\mathbf{1 4}, \mathbf{3 9}, \mathbf{4 0})$. They suggested that if the luminal drug concentration is low (sink condition), drug absorption is limited by the dissolution rate, i.e., oral absorption is improved by improving the dissolution. Conversely, if the luminal drug concentration increases and approaches saturated solubility (non-sink condition), the absorption is limited by the saturated solubility and not by the dissolution rate, i.e., improving the solubility is needed to increase the oral absorption. In rats, a shift from dissolution rate-limited to solubility-limited oral absorption of low-solubility drugs may be unlikely, even when a relatively high dose is administered orally. Some formulations have been developed to improve the dissolution 
rate, solubility, and oral absorption of lipophilic drugs (41-43). However, the improved effects are unlikely to be observed in rats due to the high concentration of bile acid. In our previous study, the dissolution of danazol was drastically improved by nanoparticulation using a novel wet-milling process, but no danazol was detected in rat plasma after oral administration of the danazol nanoparticles (44). Species differences in the dissolution and solubility of solid drugs should be considered, paticularly when rat data are used.

\section{CONCLUSIONS}

We revealed the regional and species differences in the total bile acid, phospholipid and inorganic ion concentrations, and $\mathrm{pH}$, in the rat GI tract. The regional differences in solubility of griseofulvin in each luminal fluid strongly indicated evaluation of the dissolution of drugs not only in the upper jejunum but also in the lower region of the GI tract is more important to increase the accuracy of predicting the oral absorption particularly of low-solubility drugs. Furthermore, the dissolution and oral absorption might not be readily predictable using rat data due to the higher concentration of bile acids and phospholipids in the rat as compared to humans. Although further experiments are needed to demonstrate these speculations, the information is considered to be important for effective prediction and evaluation of oral drug absorption.

\section{REFERENCES}

1. Wiedmann TS, Kamel L. Examination of the solubilization of drugs by bile salt micelles. J Pharm Sci, 2002; 91:1743-64.

2. Kleberg K, Jacobsen F, Fatouros DG, Müllertz A. Biorelevant media simulating fed state intestinal fluids: colloid phase characterization and impact on solubilization capacity. J Pharm Sci, 2010; 99:3522-32.

3. Rupp C, Steckel H, Müller BW. Solubilization of poorly water-soluble drugs by mixed micelles based on hydrogenated phosphatidylcholine. Int J Pharm, 2010; 395: 272-80.

4. Kataoka M, Masaoka Y, Sakuma S, Yamashita S. Effect of food intake on the oral absorption of poorly water-soluble drugs: in vitro assessment of drug dissolution and permeation assay system. J Pharm Sci, 2006; 95:2051-61.

5. Charman WN, Rogge MC, Boddy AW, Berger BM. Effect of food and a monoglyceride emulsion formulation on danazol bioavailability. J Clin Pharmacol, 1993; 33:381-6.

6. Overhoff KA, McConville JT, Yang W, Johnston KP, Peters JI, Williams RO 3rd. Effect of stabilizer on the maximum degree and extent of supersaturation and oral absorption of tacrolimus made by ultra-rapid freezing. Pharm Res, 2008; 25: 167-75.

7. N. Pandit, T. Trygstad, S. Croy, M. Bohorquez, and C. Koch. Effect of salts on the micellization, clouding, and solubilization behavior of pluronic F127 solutions. J Colloid Interface Sci, 2000; 222: 213-220.

8. V. V. Khutoryanskiy, G. A. Mun, Z. S. Nurkeeva, and A. V. Dubolazov. $\mathrm{pH}$ and salt effects on interpolymer complexation via hydrogen bonding in aqueous solutions. Polym Int, 2004; 53: 1382-1387.

9. Comba S, Sethi R. Stabilization of highly concentrated suspensions of iron nanoparticles using shear-thinning gels of xanthan gum. Water Res, 2009; 43: 3717-26.

10. Kimura T, Higaki K. Gastrointestinal transit and drug absorption. Biol Pharm Bull, 2002; 25:149-64.

11. Galia E, Nicolaides E, Hörter D, Löbenberg R, Reppas C, Dressman JB. Evaluation of various dissolution media for predicting in vivo performance of class I and II drugs. Pharm. Res, 1998; 15: 698-705.

12. Dressman JB, Reppas C. In vitro-in vivo correlations for lipophilic, poorly water-soluble drugs. Eur J Pharm Sci, 2000; 11: S73-80.

13. Jantratid E, Janssen N, Chokshi H, Tang K, Dressman JB. Designing biorelevant dissolution tests for lipid formulations: case example--lipid suspension of RZ-50. Eur J Pharm Biopharm, 2008; 69:776-85.

14. Takano R, Furumoto K, Shiraki K, Takata N, Hayashi Y, Aso Y, Yamashita S. Rate-limiting steps of oral absorption for poorly water-soluble drugs in dogs; prediction from a miniscale dissolution test and a physiologically-based computer simulation. Pharm Res, 2008; 25: 2334-2344.

15. Lindahl A, Ungell AL, Knutson L, Lennernäs H. Characterization of fluids from the stomach and proximal jejunum in men and women. Pharm Res, 1997; 14:497-502.

16. Pedersen BL, Müllertz A, Brøndsted H, Kristensen HG. A comparison of the solubility of danazol in human and simulated gastrointestinal fluids. Pharm Res, 2000; 17:891-4.

17. Kalantzi L, Persson E, Polentarutti B, Abrahamsson B, Goumas K, Dressman JB, Reppas C. Canine intestinal contents vs. simulated media for the assessment of solubility of two weak bases in the human small intestinal contents. Pharm Res, 2006; 23:1373-81.

18. Levet-Trafit B, Gruyer MS, Marjanovic M, Chou RC. Estimation of oral drug absorption in man based on intestine permeability in rats. Life Sci, 1996; 58:PL359-63.

19. Martignoni M, Groothuis GM, de Kanter R. Species differences between mouse, rat, dog, monkey and human CYP-mediated drug metabolism, inhibition and induction. Expert Opin Drug Metab Toxicol, 2006; 2:875-94. 
20. Bogaards JJ, Bertrand M, Jackson P, Oudshoorn MJ, Weaver RJ, van Bladeren PJ, Walther B. Determining the best animal model for human cytochrome P450 activities: a comparison of mouse, rat, rabbit, dog, micropig, monkey and man. Xenobiotica, 2000; 30:1131-52.

21. Komura H, Yasuda M, Yoshida NH, Sugiyama Y. Species difference in nisoldipine oxidation activity in the small intestine. Drug Metab Pharmacokinet, 2002; 17:427-36.

22. Scholz A, Kostewicz E, Abrahamsson B, Dressman JB. Can the USP paddle method be used to represent in-vivo hydrodynamics? J Pharm Pharmacol, 2003; 55:443-51.

23. Hagio M, Matsumoto M, Fukushima M, Hara H, Ishizuka S. Improved analysis of bile acids in tissues and intestinal contents of rats using LC/ESI-MS. J Lipid Res, 2009; 50:173-80.

24. Grefner NM, Gromova LV, Gruzdkov AA, Komissarchik IaIu. Comparative analysis of SGLT1 and GLUT2 transporter distribution in rat small intestine enterocytes and $\mathrm{Caco} 2$ cells during hexose absorption. Tsitologiia, 2010; 52:580-7.

25. Sakamoto S, Suzuki H, Kusuhara H, Sugiyama Y. Efflux mechanism of taurocholate across the rat intestinal basolateral membrane. Mol Pharm, 2006; 3:275-81.

26. Boyer JC, Campbell CE, Sigurdson WJ, Kuo SM. Polarized localization of vitamin $\mathrm{C}$ transporters, SVCT1 and SVCT2, in epithelial cells. Biochem Biophys Res Commun, 2005; 334:150-6.

27. Turner RJ, Silverman M. Sugar uptake into brush border vesicles from normal human kidney. Proc Natl Acad Sci U S A, 1977; 74:2825-9.

28. Hediger MA, Coady MJ, Ikeda TS, Wright EM. Expression cloning and cDNA sequencing of the $\mathrm{Na}+$ glucose co-transporter. Nature 1987; 330:379-81.

29. Otake K, Suzuki H, Higashi R, Yabuuchi H, Haga M, Maeda T, Cook TJ, Tamai I. Improved intestinal membrane permeability of hexose-quinoline derivatives via the hexose transporter, SGLT1. J Pharm Sci, 2008; 97:1821-30.

30. Masaoka Y, Tanaka Y, Kataoka M, Sakuma S, Yamashita S. Site of drug absorption after oral administration: assessment of membrane permeability and luminal concentration of drugs in each segment of gastrointestinal tract. Eur J Pharm Sci, 2006; 29:240-50.

31. Muraca M, Vilei MT, Miconi L, Petrin P, Antoniutti M, Pedrazzoli S. A simple method for the determination of lipid composition of human bile. J Lipid Res, 1991; 32:371-4.

32. Itami C, Taguchi R, Ikezawa H, Nakabayashi $T$. Release of ectoenzymes from small intestine brush border membranes of mice by phospholipases. Biosci Biotechnol Biochem, 1997; 61:336-40.
33. Deng JT, Hoylaerts MF, Van Hoof VO, De Broe ME. Differential release of human intestinal alkaline phosphatase in duodenal fluid and serum. Clin Chem, 1992; 38:2532-8.

34. Fotaki N, Symillides M, Reppas C. In vitro versus canine data for predicting input profiles of isosorbide-5-mononitrate from oral extended release products on a confidence interval basis. Eur J Pharm Sci, 2005; 24:115-22.

35. Jantratid E, De Maio V, Ronda E, Mattavelli V, Vertzoni M, Dressman JB. Application of biorelevant dissolution tests to the prediction of in vivo performance of diclofenac sodium from an oral modified-release pellet dosage form. Eur J Pharm Sci, 2009; 37:434-41.

36. Sugano K, Kataoka M, Mathews Cda C, Yamashita $\mathrm{S}$. Prediction of food effect by bile micelles on oral drug absorption considering free fraction in intestinal fluid. Eur J Pharm Sci, 2010; 40:118-24.

37. Kataoka M, Masaoka Y, Yamazaki Y, Sakane T, Sezaki H, Yamashita S. In vitro system to evaluate oral absorption of poorly water-soluble drugs: simultaneous analysis on dissolution and permeation of drugs. Pharm Res, 2003; 20:1674-80.

38. Okumu A, DiMaso M, Löbenberg R.. Dynamic dissolution testing to establish in vitro/in vivo correlations for montelukast sodium, a poorly soluble drug. Pharm Res, 2008; 25:2778-85.

39. Takano R, Sugano K, Higashida A, Hayashi Y, Machida M, Aso Y, Yamashita S. Oral absorption of poorly water-soluble drugs: computer simulation of fraction absorbed in humans from a miniscale dissolution test. Pharm Res, 2006; 23: 1144-56.

40. Takano R, Takata N, Saito R, Furumoto K, Higo S, Hayashi Y, Machida M, Aso Y, Yamashita S. Quantitative Analysis of the Effect of Supersaturation on in Vivo Drug Absorption. Mol Pharm, 2010; 7: 1431-1440.

41. Tanaka Y, Inkyo M, Yumoto R, Nagai J, Takano M, Nagata S. Nanoparticulation of poorly water soluble drugs using a wet-mill process and physicochemical properties of the nanopowders. Chem Pharm Bull (Tokyo), 2009; 57:1050-7.

42. Nagy ZK, Balogh A, Vajna B, Farkas A, Patyi G, Kramarics A, Marosi G.. Comparison of electrospun and extruded Soluplus ${ }^{\circledR}$-based solid dosage forms of improved dissolution. J Pharm Sci, 2012; 101:322-32.

43. Cherukuvada S, Babu NJ, Nangia A. Nitrofurantoin-p-aminobenzoic acid cocrystal: hydration stability and dissolution rate studies. J Pharm Sci, 2011; 100:3233-44.

44. Tanaka Y, Inkyo M, Yumoto R, Nagai J, Takano M, Nagata S. Evaluation of in vitro dissolution and in vivo oral absorption of drug nanopowders prepared by novel wet-milling equipment. Current Nano Science, 2010; 6: 571-576. 